\title{
Colorimetric Matching for Medical Cameras
}

\author{
Jiyoung Yoon, Cheolhwan Kim*, Shihyun Ahn \\ Kyungpook National University, Daegu Republic of Korea. \\ * Corresponding author. Tel.: +821075479656; email: kch1@knu.ac.kr \\ Manuscript submitted February 19, 2016; accepted June 18, 2016. \\ doi: 10.17706/ijcee.2016.8.3.227-233
}

\begin{abstract}
While medical equipment and IT technology advances, doctors make medical surgery using input images through cameras. They use an optical microscope or an endoscope equipped with cameras. These systems display expanded images on a monitor for the required surgical sites. It is important to display almost the same color as the actual view, in case of making surgery or observing it. If monitors display wrong color, doctors cannot accurately recognize the surgical site and have difficulty to make a surgery, and surgery observers cannot get accurate information of the surgical site. In this paper, we proposed the characterization method for medical cameras using with the ColorChecker that is commonly used to calibrate cameras and additional color values on actual surgical site. It makes the estimated coefficient through polynomial regression. It can show surgical scenes to users more precisely.
\end{abstract}

Key words: Colorimetric matching, medical camera, camera characterization, CIELAB, coefficient estimation.

\section{Introduction}

While medical equipment and IT technology advances, doctors make medical surgery using input images through cameras. They use an optical microscope or an endoscope equipped with cameras. These systems display expanded images on a monitor for the required surgical sites [1]-[4]. Fig. 1 is various use cases of imaging devices in medical procedures.
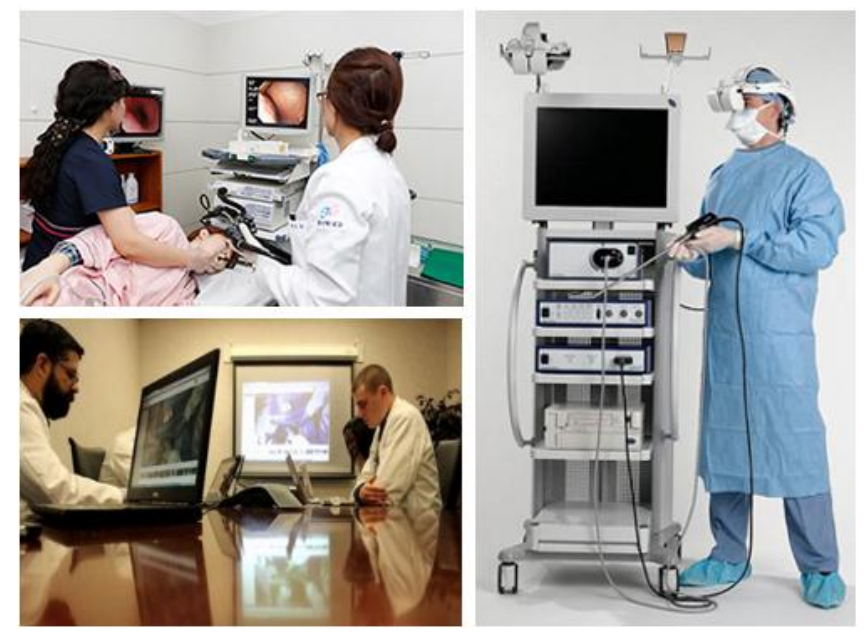

Fig. 1. Various use cases of imaging devices in medical procedures. 
It is important to display exact color of the pictures when users use medical scenes through variety display devices. If display devices show incorrect colors, then users do not recognize medical treatment position. It makes doctors cannot recognize treatment position and surgery visitors could not get correct medical scenes. Fig. 2 is the example of surgery scene display.

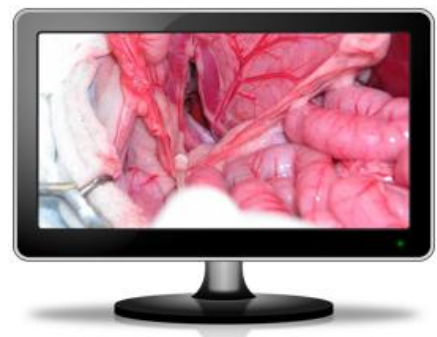

a. Correct color

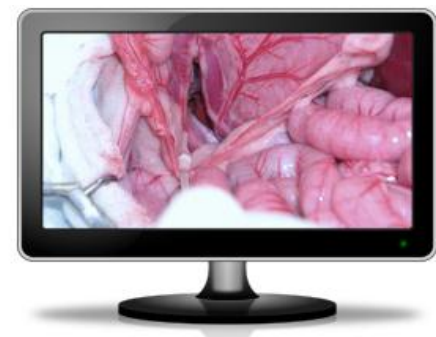

b. Incorrect color

Fig. 2. Example of surgery scene display.

In Fig. 2(a) there is displayed correct color and in Fig. 2(b) there is displayed incorrect color of surgery scene on a monitor. If a monitor display incorrect color then users may feel their eye fatigue and be hard to find surgery sites.

In this paper, we propose a new colorimetric matching method to display correct color for medical cameras and have verified it by experiments.

\section{Colorimetric Matching for Cameras}

Colorimetric matching for cameras is the relationship modeling of digital RGB [5] values from cameras and CIELAB [6] values from color meter device, which is device independent color space. It is a characterization method for cameras [7]-[10]. Fig. 3 is the process of the characterization method for cameras.

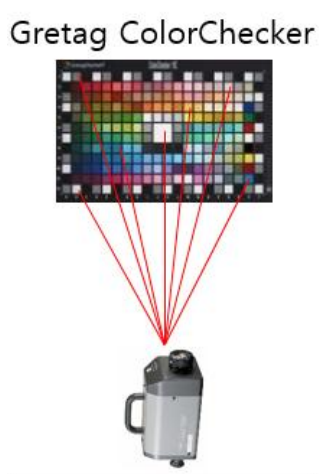

Get CIELAB values
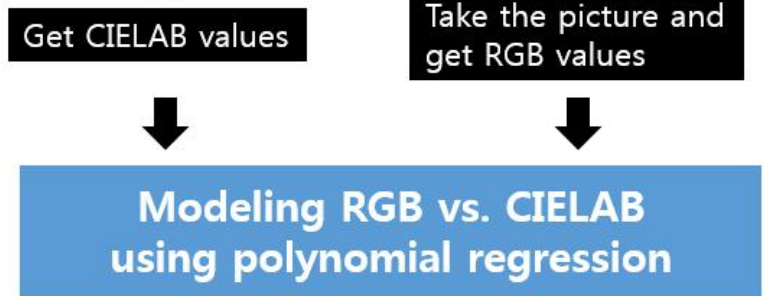

Fig. 3. Characterization method for cameras.

There is used the ColorChecker [11] for the characterization method for cameras. The ColorChecker is a color calibration target consisting of a cardboard-framed arrangement of many squares of painted samples. 
Characterization method for cameras uses CIELAB values of the ColorChecker from a color meter and RGB values from cameras. It makes the estimated coefficient through polynomial regression [12] using relationship modeling of RGB and CIELAB values. Fig. 4 is the coefficient estimation method.

$$
\begin{aligned}
L(R, G, B)= & a_{x, 0}+a_{x, 1} R+a_{x, 2} \mathrm{G}+a_{x, 3} B+a_{x, 4} R G+a_{x, 5} G B+a_{x, 6} G R \\
& +a_{x, 7} R^{2}+a_{x, 8} G^{2}+a_{x, 9} B^{2}+a_{x, 10} R G B+a_{x, 11} R^{3} \\
& +a_{x, 12} G^{3}+a_{x, 13} B^{3}+a_{x, 14} R G^{2}+a_{x, 15} R^{2} G+a_{x, 16} G B^{2} \\
& +a_{x, 17} G^{2} B+a_{x, 18} B R^{2}+a_{x, 19} B^{2} R \\
a(R, G, B)= & a_{y, 0}+a_{y, 1} R+a_{y, 2} \mathrm{G}+a_{y, 3} B+a_{y, 4} R G+a_{y, 5} G B+a_{y, 6} G R \\
& +a_{y, 7} R^{2}+a_{y, 8} G^{2}+a_{y, 9} B^{2}+a_{y, 10} R G B+a_{y, 11} R^{3} \\
& +a_{y, 12} G^{3}+a_{y, 13} B^{3}+a_{y, 14} R G^{2}+a_{y, 15} R^{2} G+a_{y, 16} G B^{2} \\
& +a_{y, 17} G^{2} B+a_{y, 18} B R^{2}+a_{y, 19} B^{2} R \\
\mathrm{~b}(R, G, B)= & a_{z, 0}+a_{z, 1} R+a_{z, 2} \mathrm{G}+a_{z, 3} B+a_{z, 4} R G+a_{z, 5} G B+a_{z, 6} G R \\
& +a_{z, 7} R^{2}+a_{z, 8} G^{2}+a_{z, 9} B^{2}+a_{z, 10} R G B+a_{z, 11} R^{3} \\
& +a_{z, 12} G^{3}+a_{z, 13} B^{3}+a_{z, 14} R G^{2}+a_{z, 15} R^{2} G+a_{z, 16} G B^{2} \\
& +a_{z, 17} G^{2} B+a_{z, 18} B R^{2}+a_{z, 19} B^{2} R
\end{aligned}
$$

Lab values

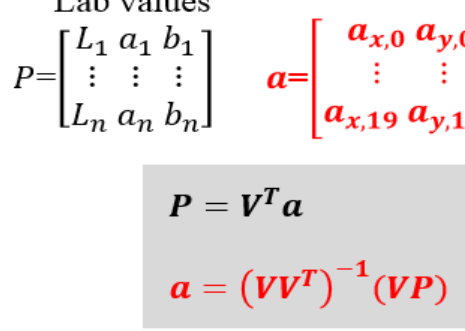

$$
\text { Input RGB values }
$$

Coefficient estimation

Fig. 4. Coefficient estimation method.

\section{Get the RGB and CILAB Values of Real Surgical Sites}

In this paper, we propose the method to display more precise colors of medical scene from cameras. It uses characterization method described in chapter 2 and additional color values on actual surgical site. The reason of using a real surgical scene is that it makes more precise color of medical scenes. To use characterization method with additional color values on actual surgical site, we propose to use the real scene of inside of human body using medical cameras. Fig. 5 is the characterization method for cameras with additional color values from the real surgical scene.

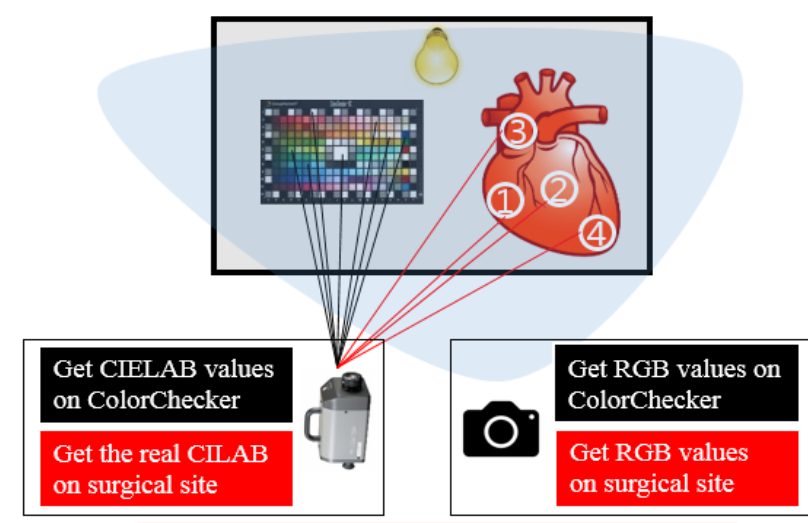

Modeling RGB vs. CIELAB

using ColorChecker and surgical site

\& Make Characterization method

using polynomial regression

Fig. 5. Characterization method for cameras with additional color values from the real surgical scene. 
The characterization method for cameras commonly uses the ColorChecker. To cover various colors, it uses custom ColorCheckers with many color samples. Our target was medical cameras. Therefore, Characterization method for medical cameras used RGB and CIELAB values from actual surgical site under the light of the surgery room. These actual surgical colors was used as additional color samples. It makes the estimated coefficient through polynomial regression. Fig. 6 is coefficient estimation method using ColorChecker and surgical site color values.

There is defined $\mathrm{P}^{\prime}$ as CIELAB values from surgical site and $\mathrm{V}^{\prime}$ as RGB values from same site. Characterization method for medical cameras estimate the specific coefficient on surgical site through polynomial regression. It calibrates the surgical scene from cameras using the specific coefficient.

$$
\begin{aligned}
\text { Lab values of ColorChecker } \\
\qquad P=\left[\begin{array}{ccc}
L_{1} & a_{1} & b_{1} \\
\vdots & \vdots & \vdots \\
L_{n} & a_{n} & b_{n}
\end{array}\right] \quad a=\left[\begin{array}{ccc}
a_{x, 0} & a_{y, 0} & a_{z, 0} \\
\vdots & \vdots & \vdots \\
a_{x, 19} & a_{y, 19} & a_{z, 19}
\end{array}\right] \\
P^{\prime}=\left[\begin{array}{ccc}
L_{1}^{\prime} & a_{1}^{\prime} & b_{1}^{\prime} \\
L_{2}^{\prime} & a_{2}^{\prime} & b_{2}^{\prime} \\
L_{3}^{\prime} & a_{3}^{\prime} & b_{3}^{\prime} \\
L_{4}^{\prime} & a_{4}^{\prime} & b_{4}^{\prime}
\end{array}\right]
\end{aligned}
$$

Lab values of surgical site

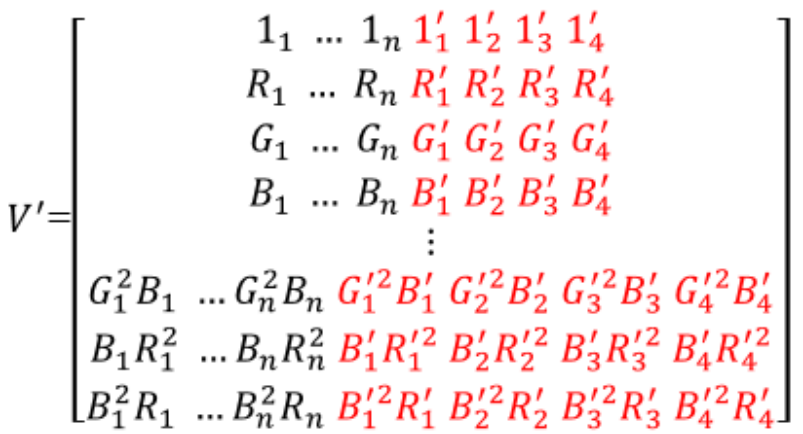

$$
\begin{aligned}
& \text { Input } R G B \text { values Input } R G B \text { values } \\
& \text { of ColorChecker of surgical site }
\end{aligned}
$$

$$
\begin{aligned}
& \boldsymbol{P}^{\prime \prime}=\left[\boldsymbol{P}, \boldsymbol{P}^{\prime}\right] \\
& \boldsymbol{P}^{\prime \prime}=\boldsymbol{V}^{\prime T} \boldsymbol{a} \\
& \boldsymbol{a}=\left(\boldsymbol{V}^{\prime} \boldsymbol{V}^{\prime T}\right)^{-1}\left(\boldsymbol{V}^{\prime} \boldsymbol{P}^{\prime \prime}\right)
\end{aligned}
$$

\section{Coefficient estimation}

Fig. 6. Coefficient estimation method using ColorChecker and surgical site color values.

\section{Experiment and Result}

\subsection{Experimental Environment}

We experimented the characterization method for medical cameras using pieces of raw meat instead of actual surgical site. We used the D65 light source that is used on surgery room. We marked test areas that are similar with blood and organ of human, and we measured RGB and CIELAB values on the marked areas using the target CCTV camera that could not take precious color of medical images and CS-200 color meter. 
CS-200 measures luminance and chromaticity. We got the 13 color samples on raw meat. We made the estimated coefficient through polynomial regression using the 13 color samples and used this estimated coefficient to make calibrated image from input images of target camera. Fig. 7 is the experimental environment.
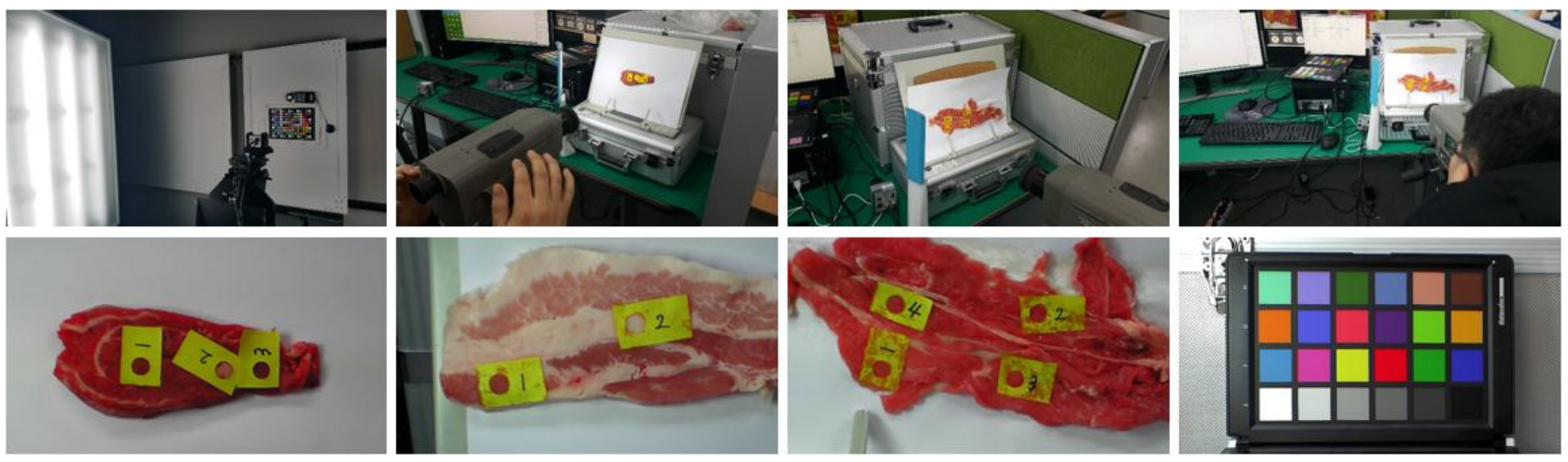

Fig. 7. Experimental environment.

\subsection{Result}

In the result of experiment, the target camera displayed more correct colors of raw meats as compared to the original image in which colorimetric matching is not applied. To compare the experiment result, we measured color variation of CIELAB three cases that are the original camera images, the calibrated camera image using only ColorChecker and the calibrated camera image using ColorChecker with additional the 13 color samples from raw meats. Table 1 is the color variation about the experiment results.

Table 1. The Color Variation about the Experiment Result

\begin{tabular}{cccc}
\hline \hline Sample Image & Original & ColorChecker & $\begin{array}{c}\text { ColorChecker \& } \\
\text { Raw meat sample }\end{array}$ \\
\hline 1 & 38.3231 & 12.4301 & 2.8439 \\
2 & 31.3020 & 8.2740 & 1.4391 \\
3 & 34.1449 & 13.2385 & 2.3305 \\
4 & 35.3909 & 15.0478 & 4.0421 \\
5 & 29.6444 & 13.3137 & 3.9762 \\
6 & 30.1825 & 13.1773 & 2.9350 \\
7 & 47.6895 & 18.1925 & 3.5113 \\
8 & 42.5826 & 16.6911 & 2.4901 \\
9 & 44.1206 & 15.9093 & 1.7043 \\
10 & 41.2341 & 14.1335 & 3.0192 \\
11 & 51.2690 & 13.1303 & 3.9057 \\
12 & 39.9688 & 8.8831 & 7.0975 \\
13 & 45.6866 & 16.1247 & 5.4379 \\
Avg & $\mathbf{3 9 . 3 4 9 2}$ & $\mathbf{1 3 . 7 3 4 3}$ & $\mathbf{3 . 4 4 1 0}$ \\
\hline \hline
\end{tabular}

As shown in Table 1, minimum color variation was measured on the case of using ColorChecker and additional color samples from raw meats. Thus medical cameras could display more realistic colors by using the estimated coefficient from the colors of actual surgical site. Fig. 8 is the result images of the experiment. 

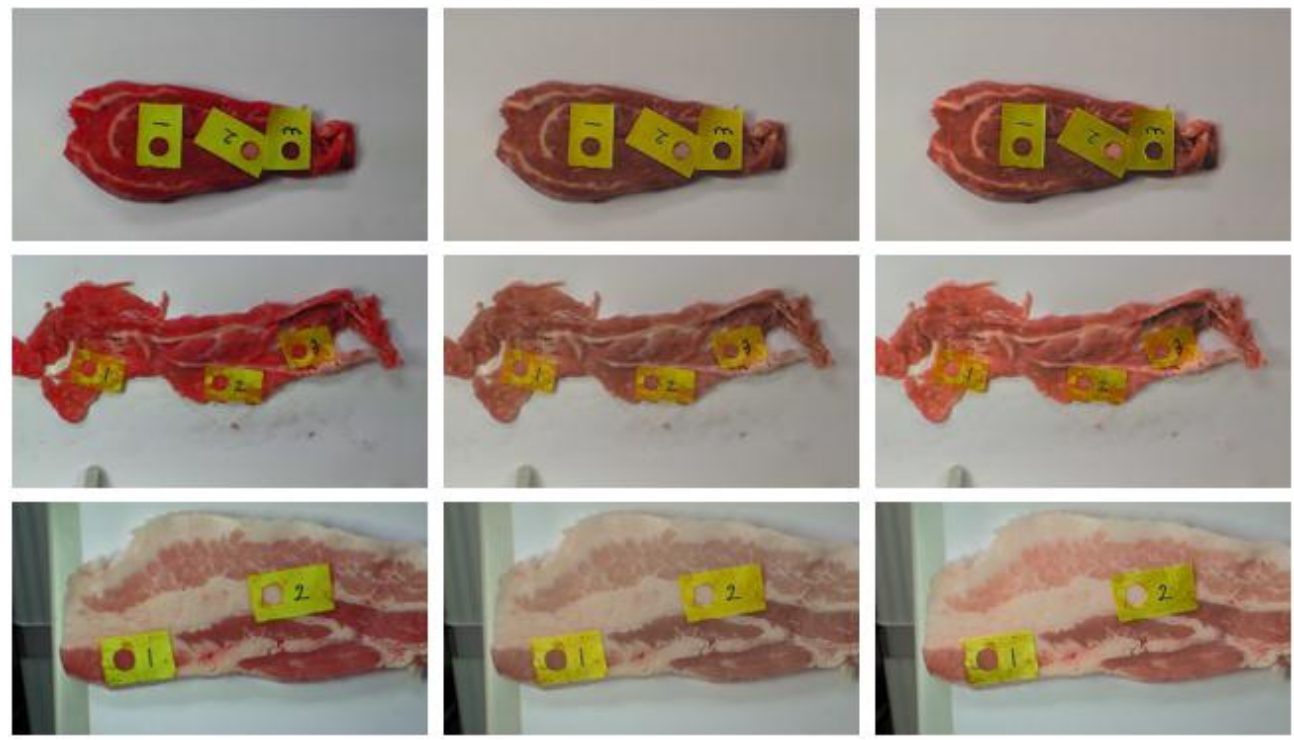

Fig. 8. Result images of the experiment. Original images (left), calibrated images with only ColorChecker (middle), and calibrated images with ColorChecker and additional colors (right) Coefficient estimation method using ColorChecker and surgical site color values.

\section{Conclusion}

In this paper, we proposed the method to display medical scene color more precisely. While medical equipment and IT technology advances, doctors make medical surgery using input images through cameras. This is an optical microscope equipped with cameras as well as the endoscope and these display expanded images on a monitor or personal display equipment for the required surgical sites. It is important to display exact color of the pictures when users use medical scenes through variety display devices. If display devices show incorrect colors, then users do not recognize medical treatment position. It bring that doctors could not recognize treatment position and surgery visitors could not get correct medical scenes. To solve this problem, we propose the characterization method for medical cameras using with the ColorChecker that is commonly used to calibrate cameras and additional color values on actual surgical site. Medical camera that is applied this method could display real colors using color values on actual surgical site.

In the future, we will get real data of human's inner skin and organs and experiment characterization method for medical camera with it. After, we will experiment characterization method for display devices.

\section{Acknowledgment}

This research was financially supported by the Ministry of Trade, Industry \& Energy (MOTIE), Korea Institute for Advancement of Technology (KIAT) and Gangwon Institute for Regional Program Evaluation (GWIRPE) through the Economic and Regional Cooperation Industry.

\section{References}

[1] Figl, M., Ede, C., et al. (2005). A fully automated calibration method for an optical see-through head mounted operating microscope with variable zoom and focus. IEEE Transaction on Medical Imaging, 1492-1499.

[2] Tamiya, T., Kawanish, M., \& Guo, S. (2011). Skull base surgery using navigation Microscope Integration system. Complex Medical Engineering, 185-187.

[3] Yoon, J. Y., Kim, H. D., \& Kim, C. (2015). Development of image error correction system for 3D optical 
microscope. Computing Technology and Information Management, 105-108.

[4] Varvaro, H., Juan-Lizandra, M. C., Alcañiz, M., Monserrat, C., Grau, V., Gil, J. A. (2001). Digital microscope with augmented reality for neurosurgery. International Congress Series, 1230, 248-253.

[5] Wikipedia Contributors. (January 8, 2016). RGB color model. Wikipedia, the Free Encyclopedia.

[6] Wikipedia Contributors. (december 29, 2015). Lab color space. Wikipedia, the Free Encyclopedia.

[7] Hong, G., Luo, M. R., \& Ronnier, P. A. (February 2001). A study of digital camera colorimetric characterization based on polynomial modeling. COLOR Research and Application, 26(1), 76-84.

[8] Giorgianni, E. J., \& Madden, T. E. (2008). Digital Color Management Encoding Solutions, pp. 51-60.

[9] Ilie, A., \& Welch, G. (Oct. 2005). Ensuring color consistency across multiple cameras. IEEE International Conference on Computer Vision (pp. 1268-1275).

[10] Park, J. S., Kim, D. W., Jang, S. W., Kim, E. S., \& Sohng, K. I. (June 2002). Digital video camera characterization considering white balance. Proceedings of IEEK Summer Conference: Vol. 25 (pp. 299-302).

[11] Wikipedia Contributors. (January 17, 2016). ColorChecker. Wikipedia, the Free Encyclopedia.

[12] Pointer, M. R., Attridge, G. G., \& Jacobson, R. E. (July 2001). Practical camera characterization for colour measurement. The Imaging Science Journal, 49(2), 63-80.

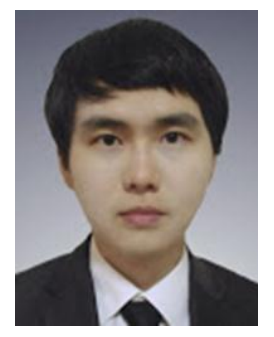

Jiyoung Yoon is currently a Ph.D. candidate in the School of Computer Science and Engineering at Kyungpook National University. His research interests include graphics standards, graphics algorithms and parallel processing. He is working with the Institute of Advanced Convergence Technology, Korea.

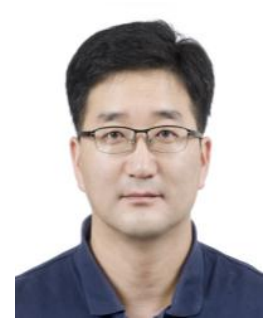

Cheolhwan Kim is currently a Ph.D. candidate in the School of Electronic, Engineering at Kyungpook National University. His research interests include computer vision, 3D reconstruction and medical image processing. He is working with the Institute of Advanced Convergence Technology, Korea.

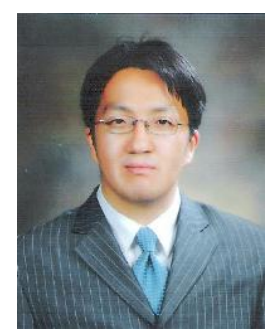

Shihyun Ahn is currently a Ph.D. candidate in the School of Electronic, Engineering at Kyungpook National University. His research interests include FPGA, computer architecture, and vision processor. He is working with the Institute of Advanced Convergence Technology, Korea. 